\title{
Asymptotic stability of a partial differential equation with an integral perturbation
}

\author{
by Katarzyna Pichór (Katowice)
}

\begin{abstract}
We study the asymptotic behaviour of the Markov semigroup generated by an integro-partial differential equation. We give new sufficient conditions for asymptotic stability of this semigroup.
\end{abstract}

1. Introduction. We study the asymptotic behaviour of the semigroup of Markov operators generated by the equation

$$
\frac{\partial u}{\partial t}+\lambda u=A u+\lambda K u
$$

where $K: L^{1}\left(\mathbb{R}^{d}\right) \rightarrow L^{1}\left(\mathbb{R}^{d}\right)$ is an integral Markov operator, $\lambda>0$ and the operator $A$ is given by

$$
A f=-\sum_{i=1}^{d} \frac{\partial\left(a_{i} f\right)}{\partial x_{i}} .
$$

If $\lambda=0$ then (1.1) is known as the Liouville equation. In this case $u(x, t)$ describes the densities of the distributions of points whose movement is governed by the differential equation

$$
x^{\prime}(t)=a(x(t)) .
$$

If we add the integral term $K$ then this movement is perturbed by random jumps of points. Then (1.1) is known as the Takacs equation and plays an important role in the theory of jump processes.

The problem of asymptotic stability of the solutions of (1.1) has been investigated by Klaczak [3], Lasota and Mackey [5], Malczak [6] and Rudnicki [9]. The main tool exploited in these papers was the Lyapunov function. In our paper we use the theory of partially-integral Markov semigroups [10]

1991 Mathematics Subject Classification: Primary 45K05, 45M05, 45M10, 47D07; Secondary 35K22, 45K05, 60J60.

Key words and phrases: integro-differential equation, Markov semigroup, asymptotic stability. 
and the concept of Hasminskii function [7, 8]. Our method allows us to strengthen the earlier results concerning the asymptotic stability of Markov semigroups (see Remark 1 and Section 4).

The organization of the paper is as follows. Section 2 contains some auxiliary definitions and results from the theory of Markov operators. The main result concerning the asymptotic stability of (1.1) is formulated in Section 3 and proved in Section 5. Section 4 contains some examples of applications.

2. Semigroup representation of solutions. Let $X \subset \mathbb{R}^{d}$ be a bounded or unbounded domain. We denote by $\partial X$ and $\bar{X}$ the boundary and the closure of $X$, respectively. We assume that the domain $X$ is normal and the boundary $\partial X$ is either empty or a surface of class $C_{\sigma}^{1}$ (see [4]).

We consider the integro-differential equation

$$
\frac{\partial u(t, x)}{\partial t}+\lambda u(t, x)=-\sum_{i=1}^{d} \frac{\partial\left(a_{i}(x) u(t, x)\right)}{\partial x_{i}}+\lambda \int_{X} k(x, y) u(t, y) d y,
$$

where $t \geq 0, x \in X$ and $\lambda$ is a positive constant. The kernel $k$ is measurable and stochastic, i.e.,

$$
\int_{X} k(x, y) d x=1 \quad \text { and } \quad k(x, y) \geq 0, \quad x, y \in X .
$$

We denote by $D$ the set of all nonnegative elements of $L^{1}(X)$ with norm one. The elements of $D$ are densities. A linear mapping $P: L^{1}(X) \rightarrow L^{1}(X)$ is called a Markov operator if $P(D) \subset D$. A semigroup $\{P(t)\}_{t \geq 0}$ of linear operators on $L^{1}(X)$ is said to be a continuous semigroup of Markov operators if $P(t)$ is a Markov operator for every $t \geq 0$ and if for every $f \in L^{1}(X)$ the function $t \mapsto P(t) f$ is continuous. A density $f_{*} \in D$ is called invariant under the semigroup $\{P(t)\}_{t \geq 0}$ if $P(t) f_{*}=f_{*}$ for every $t \geq 0$. The semigroup $\{P(t)\}_{t \geq 0}$ is called asymptotically stable if there is an invariant density $f_{*}$ such that

$$
\lim _{t \rightarrow \infty}\left\|P(t) f-f_{*}\right\|=0 \quad \text { for } f \in D .
$$

Equation (2.1) can be rewritten as the evolution equation

$$
u^{\prime}(t)=(A-\lambda I+\lambda K) u, \quad u(0) \in L^{1}(X),
$$

where $I f=f, K: L^{1}(X) \rightarrow L^{1}(X)$ is the Markov operator defined by

$$
K f(x)=\int_{X} k(x, y) f(y) d y
$$


and the operator $A$ is given by

$$
A f=-\sum_{i=1}^{d} \frac{\partial\left(a_{i} f\right)}{\partial x_{i}} .
$$

We assume that $a_{i} \in C_{\mathrm{b}}^{2}(\bar{X})$ for all $i$, where $C_{\mathrm{b}}^{k}(\bar{X})$ is the space of $k$-times differentiable bounded functions defined on $\bar{X}$ whose partial derivatives of order $\leq k$ are continuous and bounded.

Define the domain of the operator $A$ by

$$
E=\left\{f \in L^{1}(X) \cap C_{\mathrm{b}}^{1}(\bar{X}): A f \in L^{1}(X), f(x)=0 \text { for } x \in \partial X\right\} .
$$

Then we replace the operator $A$ by its closure $\bar{A}$ in $L^{1}(X)$. From now on we write $A$ for the closure $\bar{A}$.

Denote by $n(x)$ the unit inward normal vector to the boundary $\partial X$ at the point $x$. Let $a(x) \cdot n(x)$ denote the scalar product of $a(x)$ and $n(x)$. We assume that

$$
a(x) \cdot n(x) \geq 0
$$

for all $x \in \partial X$ such that the normal vector $n(x)$ is well defined. For each $\bar{x} \in X$ denote by $\pi_{t} \bar{x}$ the solution $x(t)$ of the equation

$$
x^{\prime}(t)=a(x(t))
$$

with initial condition $x(0)=\bar{x}$. From (2.4) it follows that $\pi_{t}(X) \subset X$ for all $t>0$.

The equation

$$
u^{\prime}(t)=A u
$$

generates a continuous semigroup $\{T(t)\}_{t \geq 0}$ of positive linear operators on $L^{1}(X)$. The semigroup $\{T(t)\}_{t \geq 0}$ can be given explicitly. Namely

$$
T(t) g(x)=\mathbf{1}_{X}\left(\pi_{-t} x\right) g\left(\pi_{-t} x\right) J(-t, x) \quad \text { for } g \in L^{1}(X),
$$

where $J(t, x)=\operatorname{det}(d / d x)\left[\pi_{t} x\right]$.

If $g \in E$ then the function $u(t, x)=T(t) g(x)$ is the classical solution of the equation

$$
\frac{\partial u}{\partial t}=-\sum_{i=1}^{d} \frac{\partial\left(a_{i} f\right)}{\partial x_{i}}
$$

with initial condition $u(0, x)=g(x)$ and boundary condition $u(t, x)=0$ for $x \in \partial X$ and $t \geq 0$. Since $E$ is dense in $L^{1}(X)$ and $T(t)$ is a contraction on $E$ for each $t \geq 0$, we can extend $T(t)$ in a unique way to the whole $L^{1}(X)$. This extension defines the semigroup $\{T(t)\}_{t \geq 0}$. From (2.4) and (2.6) it follows that $T(t)$ preserves integrals. Consequently, $\{T(t)\}_{t \geq 0}$ is a continuous semigroup of Markov operators. 
Let $\mathcal{A} f=A f-\lambda f+\lambda K f$. Then (2.3) can be rewritten as the evolution equation

$$
u^{\prime}(t)=\mathcal{A} u,
$$

and $u$ satisfies the initial condition $u(0)=g, g \in L^{1}(X)$. From the Phillips perturbation theorem [1], (2.7) generates a continuous semigroup $\{P(t)\}_{t \geq 0}$ of Markov operators on $L^{1}(X)$ given by

$$
P(t) g=u(t)=e^{-\lambda t} \sum_{n=0}^{\infty} \lambda^{n} T_{n}(t) g,
$$

where $T_{0}(t)=T(t)$ and

$$
T_{n+1}(t) g=\int_{0}^{t} T_{0}(t-s) K T_{n}(s) g d s, \quad n \geq 0 .
$$

3. Main result. We denote by $B(x, r)$ the closed ball in $X$ with centre $x$ and radius $r$. We say that a point $w \in X$ is $\varepsilon$-attainable from $v \in X$ if there are a positive integer $n$, a sequence $t_{1}, \ldots, t_{n}$ of positive numbers and a sequence $v_{0}, \ldots, v_{n}$ of elements of $X$ such that $v_{0}=v, v_{n}=w$ and

$$
k(x, y)>0
$$

for all $y \in B\left(\pi_{t_{i}} v_{i-1}, \varepsilon\right), x \in B\left(v_{i}, \varepsilon\right)$ and $i=1, \ldots, n$.

Let $A^{*}$ and $K^{*}$ be the linear operators given by

$$
A^{*} f(x)=\sum_{i=1}^{d} a_{i}(x) \frac{\partial f}{\partial x_{i}}(x), \quad K^{*} f(x)=\int_{X} k(y, x) f(y) d y .
$$

Denote by $C^{k}(X)$ the space of $k$-times differentiable functions defined on $X$ whose partial derivatives of order $\leq k$ are continuous. For every nonnegative $C^{1}$-function $f$ we put $\mathcal{A}^{*} f=A^{*} f-\lambda f+\lambda K^{*} f$. Then $\mathcal{A}^{*}$ is the adjoint operator of $\mathcal{A}$.

A measurable function $V: X \rightarrow[0, \infty)$ will be called approximable if there exists an increasing sequence $\left(v_{n}\right)$ of functions such that:

(a) $v_{n} \in D\left(\mathcal{A}^{*}\right)$ and $v_{n} \geq 0$ for every $n \in \mathbb{N}$,

(b) there is $c>0$ such that $v_{n}-\mathcal{A}^{*} v_{n} \geq-c$ for every $n \in \mathbb{N}$,

(c) $v_{n} \rightarrow V$ a.e.,

(d) the sequence $\left(\mathcal{A}^{*} v_{n}\right)$ is a.e. convergent.

If $\left(v_{n}\right)$ satisfies (a)-(d), then we put $\mathcal{A}^{*} V=\lim _{n \rightarrow \infty} \mathcal{A}^{*} v_{n}$. The function $\mathcal{A}^{*} V$ is measurable. One can check that the definition of $\mathcal{A}^{*} V$ does not depend on the choice of the sequence $\left(v_{n}\right)$, but this does not play any role in further considerations.

The main result of this paper is the following. 
THeOREM 1. Assume that for some approximable function $V$ and some compact subset $F_{0}$ of $X$ we have

$$
\sup _{x \notin F_{0}} \mathcal{A}^{*} V(x)<0 \quad \text { and } \quad \sup _{x \in X} \mathcal{A}^{*} V(x)<\infty .
$$

Suppose that for any two points $w, v \in X$ there exists an $\varepsilon>0$ such that any $w$ is $\varepsilon$-attainable from $v$. Moreover, assume that the kernel $k$ satisfies the following condition:

(K) for every $y_{0} \in X$ there exist $x_{0} \in X, q>0$ and $\delta>0$ such that

$$
k(x, y) \geq q \mathbf{1}_{B\left(x_{0}, \delta\right)}(x) \mathbf{1}_{B\left(y_{0}, \delta\right)}(y) .
$$

Then the semigroup $\{P(t)\}_{t \geq 0}$ is asymptotically stable.

The proof of Theorem 1 is given in Section 5. In the next section we give some examples of applications.

REMARK 1. The assumptions of Theorem 1 can be divided into two groups. The $\varepsilon$-attainability and condition (3.2) describe "transitivity" properties of the semigroup. From the assumptions on $V$ it follows that $V$ is a Hasminskii function (see Section 5). Proofs of the asymptotic stability of Markov semigroups in $[3,5,6]$ use the notion of the Lyapunov function rather than the Hasminskii function. We recall the definition of the Lyapunov function. Let $X$ be an unbounded subset of $\mathbb{R}^{d}$ and let $\{P(t)\}_{t \geq 0}$ be a Markov semigroup. A function $V: X \rightarrow[0, \infty)$ is called a Lyapunov function for the semigroup $\{P(t)\}_{t \geq 0}$ if

(1) $\lim _{\|x\| \rightarrow \infty} V(x)=\infty$,

(2) there exist $t>0, \alpha<1, \beta \in \mathbb{R}$ and a dense subset $D_{0}$ of $D$ such that

$$
\int_{X} V(x) P(t) f(x) d x \leq \alpha \int_{X} V(x) f(x) d x+\beta \quad \text { for } f \in D_{0} .
$$

The existence of a Lyapunov function and the "transitivity" conditions of Theorem 1 do not imply the asymptotic stability of the semigroup. Consider the following example.

Let $X=(0, \infty)$ and $k: X \times X \rightarrow(0, \infty)$ be given by the formula

$$
k(x, y)=\frac{2}{y} e^{-2 x / y} .
$$

Let $\{P(t)\}_{t \geq 0}$ be the Markov semigroup generated by the equation

$$
\frac{\partial u(t, x)}{\partial t}+u(t, x)=K u(t, x)
$$

where the Markov operator $K: L^{1}(X) \rightarrow L^{1}(X)$ is

$$
K f(x)=\int_{0}^{\infty} k(x, y) f(y) d y .
$$


It is easy to check that

$$
P(t) f=\sum_{n=0}^{\infty} e^{-t} \frac{t^{n}}{n !} K^{n} f .
$$

The "transitivity" properties of the semigroup $\{P(t)\}_{t \geq 0}$ follow from continuity and positivity of the kernel $k$. Since

$$
\int_{0}^{\infty} k(x, y) d x=1 \quad \text { and } \quad \int_{0}^{\infty} x k(x, y) d x=\frac{y}{2}
$$

we have

$$
\int_{0}^{\infty} x K f(x) d x=\frac{1}{2} \int_{0}^{\infty} x f(x) d x .
$$

This implies that $V(x)=x$ is a Lyapunov function for the semigroup $\{P(t)\}_{t \geq 0}$. Condition (3.3) also implies that this semigroup is not asymptotically stable.

Moreover, it is difficult to check directly condition (2) in the definition of the Lyapunov function. In some cases condition (2) can be replaced by the inequality

$$
\mathcal{A}^{*} V \leq-\gamma V+\delta, \quad \gamma>0, \delta>0
$$

(cf. [5, Ch. 11.9]). If $X=\mathbb{R}^{d}$ then (3.1) is weaker than (3.4). The use of the Hasminskii function allows us to prove the asymptotic stability if condition (3.1) holds but it is difficult or impossible to check (3.4).

\section{Examples}

EXAmple 1. Let $X=\mathbb{R}^{d}$ and assume that for every $x_{0} \in X$ there exist $q>0$ and $\delta>0$ such

$$
k(x, y) \geq q \mathbf{1}_{B\left(x_{0}, \delta\right)}(x) \mathbf{1}_{B\left(x_{0}, \delta\right)}(y) .
$$

This condition implies (3.2) and the fact that each point of $X$ is $\varepsilon$-attainable from any other point of $X$. Suppose that there exist positive constants $H$ and $M$ and a bounded set $F \subset X$ such that

$$
\begin{aligned}
& \sup \{\|a(x)\|: x \notin F\}<H, \\
& \int_{X}\|y\| k(y, x) d y \leq M \quad \text { for } x \in F, \\
& \int_{X}\|y\| k(y, x) d y<\|x\|-H / \lambda \quad \text { for } x \notin F .
\end{aligned}
$$

Let $V \in C^{1}(X)$ be such that $V(x)=\|x\|$ for $\|x\| \geq 1$. We check that $V$ is approximable. Let $\varphi$ be a $C^{1}([0, \infty))$ function such that $\varphi^{\prime}(0)=1$, 
$0 \leq \varphi(x) \leq x$ for $x \in[0,1]$ and $\varphi(x)=1 / 2$ for $x \geq 1$. We define an increasing sequence $\left(v_{n}\right)$ by

$$
v_{n}(x)= \begin{cases}V(x), & x \in B(0, n), \\ n+\varphi(V(x)-n), & x \notin B(0, n) .\end{cases}
$$

It is easy to check that the sequence $\left(v_{n}\right)$ satisfies conditions (a)-(d). Then all assumptions of Theorem 1 are satisfied and, consequently, system (2.1) is asymptotically stable.

Example 2. Let $X=(0, \infty)$. We consider the one-dimensional equation (2.1) with kernel

$$
k(x, y)= \begin{cases}\gamma b(\gamma x-y), & 0 \leq y \leq \gamma x \\ 0, & 0 \leq \gamma x<y\end{cases}
$$

where $b$ is a nonnegative function such that $\int_{0}^{\infty} b(x) d x=1$. Then $k$ satisfies $(2.2)$. In $[3,9]$ a similar equation was considered with $a=$ const. Now, we generalize the results of those papers to the case when $a \in C_{\mathrm{b}}^{2}(\bar{X})$ with $a(0) \geq 0$. Assume that $\gamma>1$ and $b(x)>0$ for every $x>0$. These conditions imply (3.2) and the fact that each point of $X$ is $\varepsilon$-attainable from any other point of $X$. Suppose that

$$
\int_{e}^{\infty} b(x) \ln x d x<\infty
$$

Now, we construct a Hasminskii function for the semigroup generated by $(2.1)$. Let $\delta \in(0,1)$ be such that

$$
\int_{0}^{\delta} b(x) d x \leq \frac{1}{3}
$$

and set

$$
c=2\left(1+\int_{e}^{\infty} b(x) \ln x d x\right)
$$

Let $V \in C^{1}([0, \infty))$ be a nonnegative function such that $V(x)=c$ for $x \in\left[0, \delta^{2}\right], V$ is decreasing in $\left[\delta^{2}, \delta\right], V(x) \leq 1$ for $x \in[\delta, e]$ and $V(x)=\ln x$ for $x \geq e$. From (4.1) it follows that $\mathcal{A}^{*} V$ is a bounded function and there exists $\varepsilon>0$ such that $\mathcal{A}^{*} V(x) \leq-\varepsilon$ for $x \in\left(0, \delta^{2}\right)$ and for sufficiently large $x$. It remains to check that $V$ is approximable. We define an increasing sequence $\left(v_{n}\right)$ by

$$
v_{n}(x)= \begin{cases}V(x), & 0 \leq x \leq n \\ V(n+\varphi(x-n)), & x>n\end{cases}
$$

where $\varphi$ is the function from Example 1. It is easy to check that $\left(v_{n}\right)$ satisfies conditions (a)-(d). 
5. Proof of Theorem 1. In the proof we use some auxiliary definitions. The support of an $f \in L^{1}(X)$ is defined up to a set of measure zero by the formula

$$
\operatorname{supp} f=\{x \in X: f(x) \neq 0\} .
$$

We say that a Markov operator $P$ spreads supports if for every measurable set $A$ with $\mu(A)<\infty$ (where $\mu$ is Lebesgue measure) and for every $f \in D$ we have

$$
\lim _{n \rightarrow \infty} \mu\left(\operatorname{supp} P^{n} f \cap A\right)=\mu(A) .
$$

We say that a Markov operator $P$ satisfies condition (I) if it can be written in the form

$$
P f(x)=\int_{X} h(x, y) f(y) d y+R f(x),
$$

where $R$ is a positive contraction on $L^{1}(X)$ and the kernel $h$ is a measurable nonnegative function such that for every $y_{0} \in X$ there exist an $\varepsilon>0$ and a measurable function $\eta \geq 0$ such that $\int \eta(x) d x>0$ and

$$
h(x, y) \geq \eta(x) \mathbf{1}_{B\left(y_{0}, \varepsilon\right)}(y) .
$$

Let $\mathcal{R}=\mathcal{R}(1, \mathcal{A})=(I-\mathcal{A})^{-1}$ be the resolvent of $\mathcal{A}$. It is easy to show that $\mathcal{R}$ is a Markov operator on $L^{1}(X)$. The operator $\mathcal{R}$ is also defined by

$$
\mathcal{R} f=\int_{0}^{\infty} e^{-t} P(t) f d t .
$$

Let $Z_{0}$ be a measurable subset of $X$ and let $V: X \rightarrow[0, \infty)$ be measurable. Define

$$
D_{V}=\left\{f \in D: \int_{X} f(x) V(x) d x<\infty\right\} .
$$

The function $V$ will be called a Hasminskii function for the semigroup $\{P(t)\}_{t \geq 0}$ and the set $Z_{0}$ if there exist $M>0$ and $\varepsilon>0$ such that for every $f \in D_{V}$ we have

$$
\begin{aligned}
\int_{X} V(x) \mathcal{R} f(x) d x \leq & \int_{X} V(x) f(x) d x+M \int_{Z_{0}} \mathcal{R} f(x) d x \\
& -\varepsilon \int_{X \backslash Z_{0}} \mathcal{R} f(x) d x .
\end{aligned}
$$

The following criterion for asymptotic stability of Markov semigroups is proved in [8].

TheOREM 2. Let $\{P(t)\}_{t \geq 0}$ be a Markov semigroup generated by (2.7) and $P=P\left(t_{0}\right)$ for some $t_{0}>0$. Assume that the operator $P$ spreads supports and satisfies (I). Let $Z_{0} \subset X$ be a compact set. Assume that there exists a 
Hasminskii function for the semigroup $\{P(t)\}_{t \geq 0}$ and the set $Z_{0}$. Then the semigroup $\{P(t)\}_{t \geq 0}$ is asymptotically stable.

The thread of the proof of Theorem 1 is as follows. First we check that for each $t_{0}>0$ the operator $P\left(t_{0}\right)$ spreads supports. This follows from the $\varepsilon$-attainability assumption. Then using (3.2) we check condition (I). And finally from (3.1) we conclude that $V$ is a Hasminskii function.

From (2.6) and (2.8) it follows that the operator $T_{n}$ for $n=1,2, \ldots$ can be written in the form

$$
T_{n}(t) f(x)=\int_{X} k_{n}(t, x, y) f(y) d y,
$$

where

$$
k_{1}(t, x, y)=\int_{0}^{t} \mathbf{1}_{X}\left(\pi_{s-t} x\right) k\left(\pi_{s-t} x, \pi_{s} y\right) J(s-t, x) d s,
$$

and for $n=2,3, \ldots$,

$$
k_{n}(t, x, y)=\int_{0}^{t} \int_{X} \mathbf{1}_{X}\left(\pi_{s-t} x\right) k\left(\pi_{s-t} x, z\right) k_{n-1}(s, z, y) J(s-t, x) d z d s .
$$

Set

$$
h(t, x, y)=e^{-\lambda t} \sum_{n=1}^{\infty} \lambda^{n} k_{n}(t, x, y) .
$$

Then from (2.8) it follows that

$$
P(t) f(x)=\int_{X} h(t, x, y) f(y) d y+e^{-\lambda t} T(t) f(x) .
$$

The semigroup property $P(t+s)=P(t) P(s)$ and (5.2) imply

$$
P(t+s) f(x) \geq \int_{X}\left(\int_{X} h(t, x, z) h(s, z, y) d z\right) f(y) d y
$$

for every $f \in D$. From (5.2), (5.3) and Theorem $\mathrm{C}$ of $[2, \mathrm{Ch} . \mathrm{V}]$ it follows that

$$
h(t+s, x, y) \geq \int_{X} h(t, x, z) h(s, z, y) d z .
$$

We divide the proof of Theorem 1 into a sequence of lemmas.

Lemma 1. Let $v, w \in X$. Assume that $w$ is $\varepsilon$-attainable from $v$ for some $\varepsilon>0$. Then there exist constants $n \geq 1, \delta>0$ and $0<\alpha<\beta$ such that

$$
k_{n}(t, x, y)>0
$$

for all $t \in(\alpha, \beta), x \in B(w, \delta)$ and $y \in B(v, \delta)$. 
$\operatorname{Proof}$. Since $w$ is $\varepsilon$-attainable from $v$ there are $n$, a sequence $t_{1}, \ldots, t_{n}$ of positive numbers and a sequence $v_{0}, \ldots, v_{n}$ of elements of $X$ such that $v_{0}=v, v_{n}=w$ and

$$
k(x, y)>0
$$

for $y \in B\left(\pi_{t_{i}} v_{i-1}, \varepsilon\right), x \in B\left(v_{i}, \varepsilon\right)$ and $i=1, \ldots, n$. Let $s_{i}=t_{1}+\ldots+t_{i}$, $i=1, \ldots, n$. First we show that there exist $\gamma_{1}>0$ and $\delta_{1}>0$ with $\delta_{1} \leq \varepsilon$ such that

$$
k_{1}(t, x, y)>0
$$

for all $x \in B\left(\pi_{t_{2}} v_{1}, \delta_{1}\right), y \in B\left(v, \delta_{1}\right), t \in\left(s_{2}, s_{2}+\gamma_{1}\right)$.

Set $\varphi(s, x)=\mathbf{1}_{X}\left(\pi_{s} x\right) J(s, x)$. Thus for $\tau>0$,

$$
\begin{aligned}
k_{1}\left(s_{2}+\tau, x, y\right) & =\int_{0}^{s_{2}+\tau} \varphi\left(s-s_{2}-\tau, x\right) k\left(\pi_{s-s_{2}-\tau} x, \pi_{s} y\right) d s \\
& \geq \int_{s_{1}}^{s_{1}+\tau} \varphi\left(s-s_{2}-\tau, x\right) k\left(\pi_{s-s_{2}-\tau} x, \pi_{s} y\right) d s .
\end{aligned}
$$

Now we use a well-known theorem on the continuous dependence of solutions of differential equations on the initial condition. Since $v_{1} \in X, \pi_{t_{2}} v_{1} \in X$, $v \in X, \pi_{t_{1}} v \in X$ there exist $\gamma_{1}>0$ and $\delta_{1}>0, \delta_{1} \leq \varepsilon$ such that

$$
\pi_{s-s_{2}-\tau} x \in B\left(v_{1}, \varepsilon\right), \quad \pi_{s} y \in B\left(\pi_{t_{1}} v, \varepsilon\right)
$$

for all $0 \leq \tau \leq \gamma_{1}, s \in\left(s_{1}, s_{1}+\tau\right), x \in B\left(\pi_{t_{2}} v_{1}, \delta_{1}\right)$ and $y \in B\left(v, \delta_{1}\right)$. This implies that

$$
k\left(\pi_{s-s_{2}-\tau} x, \pi_{s} y\right)>0 .
$$

Consequently, $k_{1}(t, x, y)>0$ for all $y \in B\left(v, \delta_{1}\right), x \in B\left(\pi_{t_{2}} v_{1}, \delta_{1}\right)$ and $t \in\left(s_{2}, s_{2}+\gamma_{1}\right)$. Analogously, by induction we can show that there exist $\gamma_{m-1}>0$ and $\delta_{m-1}>0$ with $\delta_{m-1} \leq \varepsilon$ such that

$$
k_{m-1}(t, x, y)>0
$$

for all $x \in B\left(\pi_{t_{m}} v_{m-1}, \delta_{m-1}\right), y \in B\left(v, \delta_{m-1}\right), t \in\left(s_{m}, s_{m}+\gamma_{m-1}\right)$ and $m=2, \ldots, n$.

Now we show that

$$
k_{n}(t, x, y)>0
$$

for all $x \in B\left(v_{n}, \delta_{n}\right), y \in B\left(v, \delta_{n}\right)$ and $t \in\left(s_{n}, s_{n}+\gamma_{n}\right)$. Let $\tau>0$. Then

$$
k_{n}\left(s_{n}+\tau, x, y\right)=\int_{0}^{s_{n}+\tau} \int_{X} \varphi\left(s-s_{n}-\tau, x\right) k\left(\pi_{s-s_{n}-\tau} x, z\right) k_{n-1}(s, z, y) d z d s
$$




$$
\begin{aligned}
& \geq \int_{s_{n}}^{s_{n}+\tau} \int_{B\left(\pi_{t_{n}} v_{n-1}, \delta_{n-1}\right)} \varphi\left(s-s_{n}-\tau, x\right) \\
& \quad \times k\left(\pi_{s-s_{n}-\tau} x, z\right) k_{n-1}(s, z, y) d z d s .
\end{aligned}
$$

Let $\gamma_{n}>0$ and $\delta_{n}>0$ be constants such that $\gamma_{n} \leq \gamma_{n-1}, \delta_{n} \leq \min \left\{\varepsilon, \delta_{n-1}\right\}$ and

$$
\pi_{s-s_{n}-\tau} x \in B\left(v_{n}, \varepsilon\right)
$$

for $0 \leq \tau \leq \gamma_{n}, s \in\left(s_{n}, s_{n}+\tau\right)$ and $x \in B\left(v_{n}, \delta_{n}\right)$. This implies that $k\left(\pi_{s-s_{n}-\tau} x, z\right)>0$. From (5.6) we obtain $k_{n-1}(s, z, y)>0$. Thus (5.7) holds for all $x \in B\left(v_{n}, \delta_{n}\right), y \in B\left(v, \delta_{n}\right)$ and $t \in(\alpha, \beta)$, where $\alpha=s_{n}$ and $\beta=s_{n}+\gamma_{n}$.

Lemma 2. Assume that for any two points $w, v \in X$ there exists an $\varepsilon>0$ such that $w$ is $\varepsilon$-attainable from $v$. Then for every compact set $F \subset X$ there exists $t(F)$ such that $h(t, x, y)>0$ for all $x, y \in F$ and $t \geq t(F)$, where $h$ is defined in (5.1).

Proof. First we show that for each $v \in X$ there exist $\delta>0$ and $t_{0}(v)$ such that

$$
h(t, x, y)>0 \quad \text { for } x, y \in B(v, \delta) \text { and } t \geq t_{0}(v) .
$$

Indeed, from Lemma 1 and (5.1) it follows that there exist $0<\alpha<\beta$ such that

$$
h(t, x, y)>0 \quad \text { for } x, y \in B(v, \delta) \text { and } t \in[\alpha, \beta] .
$$

Using (5.4) $n$ times we obtain

$$
h(t, x, y)>0 \quad \text { for } x, y \in B(v, \delta) \text { and } t \in[n \alpha, n \beta] .
$$

Let $n_{0}$ be a positive integer such that $\left(n_{0}+1\right) \alpha<n_{0} \beta$. Then (5.10) implies that $h(t, x, y)>0$ for all $x, y \in B(v, \delta)$ and $t \geq t_{0}(v)=n_{0} \alpha$.

From (5.4), (5.8) and Lemma 1 we deduce that for all $v, w \in X$ there exist $\delta>0$ and $t_{0}(v, w)$ such that

$$
h(t, x, y)>0 \quad \text { for } x \in B(v, \delta), y \in B(w, \delta) \text { and } t \geq t_{0}(v, w) .
$$

The assertion now follows from the compactness of $F \times F$.

Lemma 3. Assume that for any two points $w, v \in X$ there exists an $\varepsilon>0$ such that $w$ is $\varepsilon$-attainable from $v$. Then for each $t_{0}>0$ the Markov operator $P\left(t_{0}\right)$ spreads supports.

Proof. Let $P=P\left(t_{0}\right)$. Since $X$ is a metric space it is sufficient to check that for every compact set $F$ and every density $f$,

$$
\mu\left(\operatorname{supp} P^{n} f \cap F\right)=\mu(F)
$$


for sufficiently large $n$. From Lemma 2 it follows that for every $f \in D$ there exists $t_{1}>0$ such that for each $t \geq t_{1}$,

$$
\mu(\operatorname{supp} P(t) f \cap F)=\mu(F) .
$$

This implies that (5.12) holds for all $n>t_{1} / t_{0}$.

Lemma 4. Assume that condition $(\mathrm{K})$ holds. Then for some $t_{0}$ the operator $P\left(t_{0}\right)$ satisfies condition $(\mathrm{I})$.

Pr o of. Fix $y_{0} \in X$ and assume that $x_{0}, q$ and $\delta$ satisfy (3.2). Then there exist $\tau, \varepsilon>0$ such that for every $|t| \leq \tau$ we have

$$
\pi_{t}\left(B\left(x_{0}, \varepsilon\right)\right) \subset B\left(x_{0}, \delta\right) \text { and } \pi_{t}\left(B\left(y_{0}, \varepsilon\right)\right) \subset B\left(y_{0}, \delta\right) .
$$

Fix $t_{0} \in(0, \tau)$ and set

$$
\eta(x)=\lambda e^{-\lambda t_{0}} q \int_{0}^{t_{0}} \psi(s, x) \mathbf{1}_{B\left(x_{0}, \delta\right)}\left(\pi_{s-t_{0}} x\right) d s,
$$

where $\psi(s, x)=\mathbf{1}_{X}\left(\pi_{s-t_{0}} x\right) J\left(s-t_{0}, x\right)$. Then from (5.13) it follows that

$$
\int_{X} \eta(x) d x \geq \int_{B\left(x_{0}, \varepsilon\right)} \eta(x) d x \geq \lambda e^{-\lambda t_{0}} q \int_{0}^{t_{0}} \int_{B\left(x_{0}, \varepsilon\right)} \psi(s, x) d x d s>0 .
$$

Now we check that $P\left(t_{0}\right)$ satisfies (I). Indeed,

$$
\begin{aligned}
h\left(t_{0}, x, y\right) & \geq \lambda e^{-\lambda t_{0}} k_{1}\left(t_{0}, x, y\right)=\lambda e^{-\lambda t_{0}} \int_{0}^{t_{0}} \psi(s, x) k\left(\pi_{s-t_{0}} x, \pi_{s} y\right) d s \\
& \geq \lambda e^{-\lambda t_{0}} \int_{0}^{t_{0}} q \psi(s, x) \mathbf{1}_{B\left(x_{0}, \delta\right)}\left(\pi_{s-t_{0}} x\right) \mathbf{1}_{B\left(y_{0}, \delta\right)}\left(\pi_{s} y\right) d s,
\end{aligned}
$$

and, consequently, $h\left(t_{0}, x, y\right) \geq \eta(x) \mathbf{1}_{B\left(y_{0}, \varepsilon\right)}(y)$, which completes the proof.

We check that $V$ is a Hasminskii function.

Lemma 5. Assume that there exists an approximable function $V$ and $a$ compact set $F_{0} \subset X$ such that

$$
\sup _{x \notin F_{0}} \mathcal{A}^{*} V(x)<0 \quad \text { and } \quad \sup _{x \in X} \mathcal{A}^{*} V(x)<\infty .
$$

Then $V$ is a Hasminskii function for the semigroup $\{P(t)\}_{t \geq 0}$ and the set $F_{0}$.

Proof. Let $M$ and $\varepsilon$ be positive constants such that $\mathcal{A}^{*} V(x)<M$ for all $x \in X$ and $\mathcal{A}^{*} V(x)<-\varepsilon$ for $x \notin F_{0}$. It is sufficient to check the inequality

$$
\begin{array}{rl}
\int_{X} V(x) \mathcal{R} & f(x) d x \\
\leq & \int_{X} V(x) f(x) d x+\int_{X} \mathcal{A}^{*} V(x) \mathcal{R} f(x) d x \quad \text { for } f \in D_{V},
\end{array}
$$


which can be rewritten as

$$
\int_{X}\left(V(x)-\mathcal{A}^{*} V(x)\right) \mathcal{R} f(x) d x \leq \int_{X} V(x) f(x) d x .
$$

Since $V$ is approximable we can find an increasing sequence $\left(v_{n}\right)$ which satisfies conditions (a)-(d). From the Lebesgue monotone convergence theorem,

$$
\lim _{n \rightarrow \infty} \int_{X} v_{n}(x) f(x) d x=\int_{X} V(x) f(x) d x .
$$

The sequence $\left(v_{n}-\mathcal{A}^{*} v_{n}\right)$ is a.e. convergent to $V-\mathcal{A}^{*} V$. Since $v_{n}-\mathcal{A}^{*} v_{n} \geq-c$ we have $\left(v_{n}-\mathcal{A}^{*} v_{n}\right) \mathcal{R} f \geq-c \mathcal{R} f$. From the Fatou lemma we obtain

$$
\int_{X}\left(V(x)-\mathcal{A}^{*} V(x)\right) \mathcal{R} f(x) d x \leq \liminf _{n \rightarrow \infty} \int_{X}\left(v_{n}(x)-\mathcal{A}^{*} v_{n}(x)\right) \mathcal{R} f(x) d x .
$$

As $v_{n} \in D\left(\mathcal{A}^{*}\right)$ we have

$$
\begin{aligned}
\int_{X}\left(v_{n}(x)-\mathcal{A}^{*} v_{n}(x)\right) \mathcal{R} f(x) d x & =\int_{X} v_{n}(x)(I-\mathcal{A}) \mathcal{R} f(x) d x \\
& =\int_{X} v_{n}(x) f(x) d x
\end{aligned}
$$

and, consequently,

$$
\int_{X}\left(V(x)-\mathcal{A}^{*} V(x)\right) \mathcal{R} f(x) d x \leq \lim _{n \rightarrow \infty} \int_{X} v_{n}(x) f(x) d x=\int_{X} V(x) f(x) d x .
$$

\section{References}

[1] N. Dunford and J. T. Schwartz, Linear Operators, Part I, Interscience, New York, 1968.

[2] S. R. Foguel, The Ergodic Theory of Markov Processes, Van Nostrand Reinhold, New York, 1969.

[3] J. Klaczak, Stability of a transport equation, Ann. Polon. Math. 49 (1988), 69-80.

[4] M. Krzyżański, Partial Differential Equations of Second Order, Vol. I, PWN, Warszawa, 1971.

[5] A. Lasota and M. C. Mackey, Chaos, Fractals and Noise. Stochastic Aspects of Dynamics, Appl. Math. Sci. 97, Springer, New York, 1994.

[6] J. Malczak, Weak and strong convergence of $L^{1}$ solutions of a transport equation, Bull. Polish. Acad. Sci. Math. 40 (1992), 59-72.

[7] K. Pichór and R. Rudnicki, Asymptotic behaviour of Markov semigroups and applications to transport equations, ibid. 45 (1997), 379-397.

[8] —, 一, Stability of Markov semigroups and applications to parabolic systems, J. Math. Anal. Appl., to appear.

[9] R. Rudnicki, Asymptotic behaviour of a transport equation, Ann. Polon. Math. 57 (1992), 45-55. 
[10] R. Rudnicki, On asymptotic stability and sweeping for Markov operators, Bull. Polish Acad. Sci. Math. 43 (1995), 245-262.

Institute of Mathematics

Silesian University

Bankowa 14

40-007 Katowice, Poland

E-mail: pichor@ux2.math.us.edu.pl

Reçu par la Rédaction le 13.5.1997

Révisé le 8.7.1997 